This document is the Accepted Manuscript version of a Published Work that appeared in final form in The Journal of Physical Chemistry B, copyright (C) American Chemical Society after peer review and technical editing by publisher. To access the final edited and published work see

https://dx.doi.org/10.1021/acs.jpcb.7b08414

\title{
Characterization of the Distribution of Pyrene Molecules in Confined Geometries with the Model Free Analysis
}

\author{
Xinzhi Cao, Remi Casier, Hunter Little, Jean Duhamel* \\ Institute for Polymer Research, Waterloo Institute for Nanotechnology, Department of \\ Chemistry, University of Waterloo, Waterloo, ON N2L 3G1, Canada
}

* To Whom correspondence should be addressed. 


\section{ABSTRACT}

Evidence is provided showing that global Model Free Analysis (MFA) of monomer and excimer fluorescence decays of pyrene dissolved in aqueous solutions of sodium dodecyl sulfate (SDS) provides the same structural and dynamic information on SDS micelles as the well-established Micelle Model (MM) does. Both MFA and MM were employed to characterize the quenching kinetics between dyes and quenchers located in surfactant micelles and the aggregation number of surfactant micelles. However, contrary to the MM which assumes that dyes and quenchers distribute themselves among SDS micelles according to a Poisson distribution and react with a rate constant that is proportional to the number of reactants in a micelle, the MFA accomplishes this task without making any assumption about the process of pyrene excimer formation in SDS micelles. The ability of the MFA to retrieve accurately the molar fraction of pyrene molecules that are isolated in SDS micelles and do not form excimer was taken advantage of to establish that it equaled the Poisson probability of exciting micelles that contained a single pyrene. The molar fraction of isolated pyrenes could then be utilized to determine the aggregation number of the SDS micelles, and the rate constant of excimer formation between one excited and one ground-state pyrene located inside a same micelle. Within experimental error, both the MFA and MM yielded the same micelle aggregation number and rate constant of excimer formation, with the MFA making no prior assumptions about the physical principles underlying the process of excimer formation contrary to the MM. The ability of the MFA to retrieve quantitative parameters providing structural and dynamic information about macromolecular systems with no prior knowledge about their architecture or labeling scheme implies that it can be applied to characterize a wide range of macromolecular architectures in solution. 


\section{INTRODUCTION}

Over the years, fluorescence quenching experiments have provided invaluable structural and dynamic information about macromolecules and their supramolecular assemblies. ${ }^{1}$ In these experiments, dyes and quenchers are introduced within a few nanometers of each other inside the macromolecular system, close enough for them to communicate with each other by some photophysical pathway such as fluorescence resonance energy transfer (FRET), ${ }^{2,3}$ electron transfer (ET), ${ }^{3,4}$ or pyrene excimer fluorescence (PEF), ${ }^{5,6}$ to name but a few. The macromolecules that are overwhelmingly targeted by the scientific community are those that can be labeled at one specific position with the dye and at another specific position with the quencher, typically at the chain ends of a linear oligomer, with a well-known chain length separating the dye from the quencher. Such conditions result in a single quenching rate constant which can be easily extracted through a simple sum-of-exponentials analysis of the fluorescence decay of the dye. ${ }^{5,7}$ These experiments take full advantage of the equivalence that exists between the number of quenching rate constants and the number of chain lengths spanning every pair of dye and quencher, a single chain length resulting in a single quenching rate constant. ${ }^{8}$

Unfortunately, while this procedure is ideally suited to characterize oligomers such as oligopeptides or oligonucleotides which are monodisperse in length, labeled specifically at the chain ends, and short enough to enable proper photophysical communication between the two dyes, it fails for macromolecules that do not lend themselves to this specific labeling scheme whereby one dye and one quencher are attached at two (and only two) specific positions of the macromolecule that are separated by just a few nanometers. ${ }^{6,7}$ Examples of macromolecules that do not obey these conditions include any polymer with a degree of polymerization greater than 100 and whose end-to-end distance is larger than a few nanometers or dendrimers with multiple 
identical terminals. Typical labeling schemes for such macromolecules would randomly target residues along the backbone of polymers or the reactive terminal ends of dendrimers. The main consequence of these labeling schemes is to introduce a distribution of dye-quencher distances that results in a distribution of quenching rate constants that must be accounted for mathematically in order to retrieve dynamic or structural information about the fluorescently labeled macromolecules under study from the analysis of their fluorescence decays. Unfortunately synthetic chemists have been very successful at generating novel macromolecular architectures and modellers have lagged far behind in the implementation of mathematical models that would handle the complex quenching kinetics between dyes and quenchers attached at more than two specific positions on these ever more complex macromolecules. The extent of complexity that can now be achieved for macromolecular architectures has been captured by Stals et al. when they asked in the title of their publication "how far can we push polymer architectures?" 9 The lack of scientific interest in developing new analysis models to study fluorescent macromolecules in solution by fluorescence techniques is apparent from the fact that the only noticeable advancement made over the past 20 years to develop new models for the analysis of fluorescence decays acquired with macromolecules bearing more than one dye and one quencher has been the establishment of the fluorescence blob model (FBM) which handles macromolecules randomly labeled with pyrene and whose internal dynamics were characterized through PEF analysis. ${ }^{6,710,11}$

The derivation of the FBM followed the traditional approach for developing models that would fit the fluorescence decays of families of macromolecules labeled according to a specific reaction scheme (random labeling of linear chains with pyrene for the FBM). The traditional approach aims to capture the main physical features of the quenching process resulting from the labeling scheme applied to a given macromolecule and these features are then translated into 
mathematical equations that are used to create a program to fit the decays of the fluorescently labeled macromolecules. The problem with this traditional approach is that the implementation of a new model typically takes years-to-decades to implement and reach widespread agreement in the scientific community while synthetic chemists constantly come up with novel macromolecular architectures which, ideally, would need to be characterized in solution by fluorescence. This discussion highlights the disconnect that currently exists between the large number of macromolecular architectures that have been created over the years ${ }^{9}$ and the dearth of suitable models to study them quantitatively by fluorescence. It begs for a departure from the traditional approach toward the development of models that are specific for a single type of macromolecular architecture to a more flexible one that could handle a variety of macromolecules.

The model free analysis (MFA) was introduced 12 years ago to address this situation. ${ }^{12}$ As its name implies, the MFA makes no assumption about the quenching mechanism and thus can, in principle, be applied to any type of macromolecular architecture that has been labeled with pyrene. ${ }^{6,12,13}$ The MFA is so versatile that it not only applies to characterize the behaviour of pyrene-labeled macromolecules (PyLMs) in solution through the analysis of intramolecular pyrene excimer formation, but also that of pyrene monolabeled peptidic antibiotics embedded in a lipid membrane forming excimer intermolecularly. ${ }^{14}$ The present study further expands the realm of application of the MFA by demonstrating that the MFA applied to the analysis of fluorescence decays of SDS aqueous solutions containing different pyrene concentrations yielded the same dynamic and structural information about surfactant micelles as that obtained from an analysis based on the classic Micelle Model (MM). Pyrene has often been used to characterize the interactions of surfactant micelles with other compounds such as nucleotide 5'monophosphate, ${ }^{15,16}$ bile salts, ${ }^{17}$ or proteins ${ }^{18}$ Furthermore, time-resolved fluorescence of pyrene 
in surfactant micelles is known to yield more accurate information on the aggregation number of surfactant micelles compared to steady-state measurements. ${ }^{19}$

In the present study, micellar solutions of $50 \mathrm{mM}$ sodium dodecyl sulfate (SDS) in water were prepared with pyrene concentrations of up to $0.7 \mathrm{mM}$. The time-resolved fluorescence decays of the pyrene monomer and excimer were acquired at each pyrene concentration. They were analyzed globally according to the MFA and MM. The MM yielded the parameters $k_{\mathrm{q}}$ and $<n>$ which are the rate constant of pyrene excimer formation in an SDS micelle containing one groundstate and one excited pyrene and the average number of pyrenes per micelle. The MM parameters $k_{\mathrm{q}}$ and $\left\langle n>\right.$ were found to be directly related to the parameters $\left\langle k>\right.$ and $f_{\text {Mfree }}$ obtained from MFA of the decays, which are the average rate constant of pyrene excimer formation inside a micelle and the molar fraction of pyrene molecules that are single occupants of an SDS micelle, respectively. In particular, $\langle n\rangle$ was found to equal $-\operatorname{Ln}\left(f_{\text {Mfree }}\right)$ so that both $\langle n\rangle$ and $f_{\text {Mfree }}$ obtained from, respectively, the MM and the MFA could be used interchangeably to determine $N_{\text {agg }}$, the number of SDS molecules constituting an SDS micelle. In so doing, this study established that the MFA provides the same dynamic and structural information about SDS micelles as the MM, but whereas the applicability of the MM is solely limited to the study of surfactant micelles, the MFA can be applied to any system forming excimer by dynamic encounters between two pyrene labels. It highlights the impressive analytical capabilities of the MFA and supports the claim made earlier that it can be applied to a remarkably varied number of macromolecular systems displaying complex kinetics of pyrene excimer formation without prior knowledge about the process of pyrene excimer formation and the distribution of the pyrene labels in the system being investigated. ${ }^{6,13}$ 


\section{EXPERIMENTAL}

Chemicals: Pyrene, SDS, and $\mathrm{NaCl}$ were purchased from Sigma-Aldrich. Before fluorescence measurements, pyrene was purified by 4-5 crystallizations in methanol. The pyrene crystals were dissolved by heating methanol and after dissolution, the pyrene solution in methanol was slowly cooled to room temperature before being kept at $-15^{\circ} \mathrm{C}$ for 30 minutes. The pyrene crystals were filtered out and dried overnight under vacuum. The purity of the crystallized pyrene was assessed by obtaining a good fit of the fluorescence decay for a $2.5 \times 10^{-6} \mathrm{M}$ pyrene solution in degassed tetrahydrofuran with a monoexponential function. All aqueous solutions were prepared with deionized water (DIW) obtained from a Biopure Series 4400 Single Pass Reverse Osmosis system with a resistivity of $18 \mathrm{M} \Omega . \mathrm{cm}$.

Preparation of pyrene in aqueous SDS solutions: Stock solutions of pyrene $(0.045 \mathrm{M})$ in tetrahydrofuran (THF), SDS (0.1 M) in DIW, and $\mathrm{NaCl}(1.0 \mathrm{M})$ in DIW were prepared. A desired amount of the $0.045 \mathrm{M}$ stock solution of pyrene in THF was deposited into a $7 \mathrm{~mL}$ vial. The THF was evaporated under a gentle flow of nitrogen, leaving behind a film of pyrene. Next, the SDS and $\mathrm{NaCl}$ stock solutions were added to the vial. After vortexing the mixture for 1 minute, DIW was used to top up the volume to reach the desired concentration. Each solution was continuously sonicated for 48 hours at $45^{\circ} \mathrm{C}$ to achieve complete dissolution of pyrene.

UV-Vis spectrophotometer: All absorption spectra were acquired with a Cary 100 Bio UV-visible spectrophotometer. The molar absorption coefficient of pyrene in SDS micelles at $336 \mathrm{~nm}$ was found to equal $33,000( \pm 800) \mathrm{M}^{-1} \cdot \mathrm{cm}^{-1}$ for solutions containing $0.2,0.3$, and $0.4 \mathrm{M} \mathrm{NaCl}$. It was used to determine the pyrene concentration of all aqueous solutions with salt studied by fluorescence. 
Steady-state fluorescence: A PTI steady-state fluorometer was employed to acquire the fluorescence spectra of aqueous SDS solutions containing varied concentrations of pyrene. The aerated solutions were excited at $336 \mathrm{~nm}$ and the fluorescence signal was monitored from 350 to $600 \mathrm{~nm}$ using the front-face geometry to minimize the inner filter effect. Information about the polarity of the environment of pyrene was obtained from the fluorescence intensity ratio $I_{1} / I_{3}$ of the first to the third peak in the monomer fluorescence spectrum.

Time-resolved fluorescence decays: The monomer and excimer fluorescence decays were acquired on an IBH time-resolved fluorometer using a 340-nanoLED as the excitation source. During decay acquisition, the excitation monochromator was set at $336 \mathrm{~nm}$ while the emission monochromator was set at $375 \mathrm{~nm}$ with a $370 \mathrm{~nm}$ cut-off filter to eliminate stray light from reaching the detector and at $510 \mathrm{~nm}$ with a $480 \mathrm{~nm}$ cut-off filter for the monomer and excimer, respectively. The monomer and excimer decays spanned 1024 channels with a time-per-channel of 2.04 and 1.02 ns/ch and were acquired with 20,000 counts at the decay maximum with a repetition rate of 500 $\mathrm{kHz}$ and $1 \mathrm{MHz}$, respectively.

Fluorescence decay analysis: The monomer decays were first fitted with Equation 1 which corresponds to the Micelle Model where $\tau_{\mathrm{M}}$ is the natural lifetime of pyrene in SDS micelles, and $<n>$ and $k_{\mathrm{q}}$ were already described in the Introduction. Since PEF is applied in these experiments, the use of $\langle n\rangle$ instead of $\langle n\rangle+1$ as the average number of pyrenes per micelle in Equation 1 might appear surprising at first glance since each micelle must already contain one excited pyrene to ensure fluorescence detection. The derivation of Equation 1 for pyrenes distributed in micelles takes into account that excitation of a micelle containing $i$ pyrenes is proportional to the product of the probability of having $i$ pyrenes per micelle times $i$. The derivation can be found in SI and results in the same equation as the one derived for the Micelle Model where the dye and quenchers 
are different molecules. The program used to fit the monomer decays only was referred to as miscatbbg.

$$
\left[P y^{*}\right]=\left[P y^{*}\right]_{o} \times \exp \left[-t / \tau_{M}-<n>\left(1-\exp \left(-k_{q} t\right)\right)\right]
$$

Since the MFA fits the monomer and excimer fluorescence decays globally, Equation S5 was derived in Supporting Information (SI) for the excimer decays by assuming a Poisson distribution of the pyrene molecules in the micelles and that excimer formation occurred with a rate constant $k_{\mathrm{q}}$ multiplied by the number of ground-state pyrenes in a micelle. The program used to fit the monomer and excimer decays globally with Equations 1 and S5 according to the Micelle Model was called globmis10ebg.

The fluorescence decays were also fitted according to the MFA whose derivation has been described in details in earlier publications. ${ }^{6,12,13}$ Briefly, the MFA acknowledges that any fluorescence decay of a pyrene monomer capable of forming excimer by diffusion can be fitted by a sum-of-exponentials as shown in Equation 2.

$$
\left[P y^{*}\right]=\left[P y_{\text {diff }}^{*}\right]_{o} \times \sum_{i=1}^{n} a_{i} e^{-t / \tau_{i}}+\left[P y_{\text {free }}^{*}\right]_{o} \times e^{-t / \tau_{M}}
$$

In Equation 2, $\left[P y_{\text {diff }}{ }^{*}\right]_{\mathrm{o}}$ and $\left[P y_{\mathrm{free}}{ }^{*}\right]_{\mathrm{o}}$ represent the concentrations of pyrenes that form excimer by dynamic encounters and that do not form excimer and behave as if they were free in solution, respectively, $a_{\mathrm{i}}$ and $\tau_{\mathrm{i}}$ are the normalized pre-exponential factors $\left(\Sigma a_{\mathrm{i}}=1\right)$ and decay times that describe excimer formation by dynamic encounters, and $\tau_{\mathrm{M}}$ is the monomer lifetime. Because the 
kinetics of pyrene excimer formation are also reflected in the excimer decay, a time-dependent expression for the excimer concentration could be derived as shown in Equation S6 in SI that uses two excimer species, namely $E 0^{*}$ and $E L^{*}$ which are the excimer resulting from two properly and improperly stacked pyrene moieties emitting with lifetimes $\tau_{\mathrm{E} 0}$ and $\tau_{\mathrm{EL}}$, respectively. The use of two excimer species in the decay analysis is often necessary when the excimer is formed in restricted geometries as is often the case with PyLMs ${ }^{6,13}$ or inside surfactant micelles. ${ }^{14}$ Although the use of two discrete excimer species resulted in good fits of the fluorescence decays, by no means did this analysis prove that only two distinct pyrene excimer species were present in the SDS micelles. More excimer types might very well be present in the solutions, but their discrete distribution could not be accounted for by our coarse analysis. Nevertheless, the MFA with two discrete excimer species resulted in good fits of the fluorescence decays that yielded parameters that described the process of pyrene excimer formation in as accurate a manner as the MM.

Global MFA of the monomer and excimer fluorescence decays enables one to retrieve the pre-exponential factors $a_{\mathrm{i}}$ and decay times $\tau_{\mathrm{i}}$ in Equation 2 with excellent accuracy as well as the molar fractions $f_{\text {free }}, f_{\text {diff, }} f_{\mathrm{E} 0}$, and $f_{\mathrm{EL}}$ of all the pyrene species $P y_{\mathrm{free}}{ }^{*}, P y_{\mathrm{diff}}{ }^{*}, E 0^{*}$, and $E L^{*}$ present in the solution, respectively. ${ }^{6,11-13}$ The $a_{\mathrm{i}}$ and $\tau_{\mathrm{i}}$ parameters can then be combined to yield the average rate constant $<k>$ of pyrene excimer formation as shown in Equation 3.

$$
<k>=\left(\sum_{i=1}^{n} a_{i} \tau_{i}\right)^{-1}-\tau_{M}^{-1}
$$

What the MFA loses in molecular details about the description of the pyrene excimer formation process that would otherwise be available with more physical analyses, it gains in 
generality by providing robust information about the internal dynamics and state of all pyrene species for any PyLMs. ${ }^{6,13}$ The MFA was conducted with the program sumegs34bg which used two floating decay times (i.e. $n=2$ in Equation 2) and fitted the monomer and excimer decays globally with Equations 2 and S6, respectively.

All three analysis programs used a floating $\tau_{\mathrm{M}}$ value that remained constant within experimental error at a given salt concentration. The programs globmis10ebg and sumegs $34 \mathrm{bg}$ needed the two excimer species $E 0^{*}$ and $E L^{*}$ to obtain good fits. The parameters retrieved from the decay analyses according to the programs miscatbbg, globmis10ebg, and sumegs $34 \mathrm{bg}$ were optimized with the Marquardt-Levenberg algorithm ${ }^{20}$ and they are listed in Tables S1-6 in SI. As always done by this laboratory, both pre-exponential factors and decay times were used to optimize the kinetic parameters in the global analyses of the monomer and excimer fluorescence decays.

\section{RESULTS AND DISCUSSION}

The fluorescence spectra of pyrene in micellar solutions of $50 \mathrm{mM}$ SDS in water were acquired for different $\mathrm{NaCl}$ concentrations. They are shown in Figure 1 for the solutions without salt. The $I_{1} / I_{3}$ ratio corresponding to the fluorescence intensity ratio of the first over the third fluorescence peak of the monomer fluorescence spectrum equaled $1.03 \pm 0.04$ in agreement with the value of 1.01 reported for aqueous solutions of pyrene in SDS micelles. ${ }^{21-23}$ The fluorescence intensity of the pyrene excimer increased with increasing pyrene concentration as the SDS micelles were occupied by a larger number of pyrene molecules that promoted pyrene encounters and thus pyrene excimer formation. The behaviour observed in Figure 1A matched the reported behaviour for the interactions of pyrene molecules distributed among surfactant micelles. ${ }^{14}$ 
The monomer and excimer fluorescence decays of each solution were acquired and fitted with the analysis programs miscatbbg (Micelle Model for the monomer only), globmis10ebg (global Micelle Model analysis of the monomer and excimer decays), or sumegs34bg (global MFA of the monomer and excimer decays). All decay analyses yielded excellent fits with $\chi^{2}$ smaller than 1.30 and residuals and autocorrelation of the residuals that were randomly distributed around zero. An example of the quality of the fits with globmis10ebg is shown in Figures $1 \mathrm{~B}$ and $\mathrm{C}$ for the monomer and excimer decays, respectively.

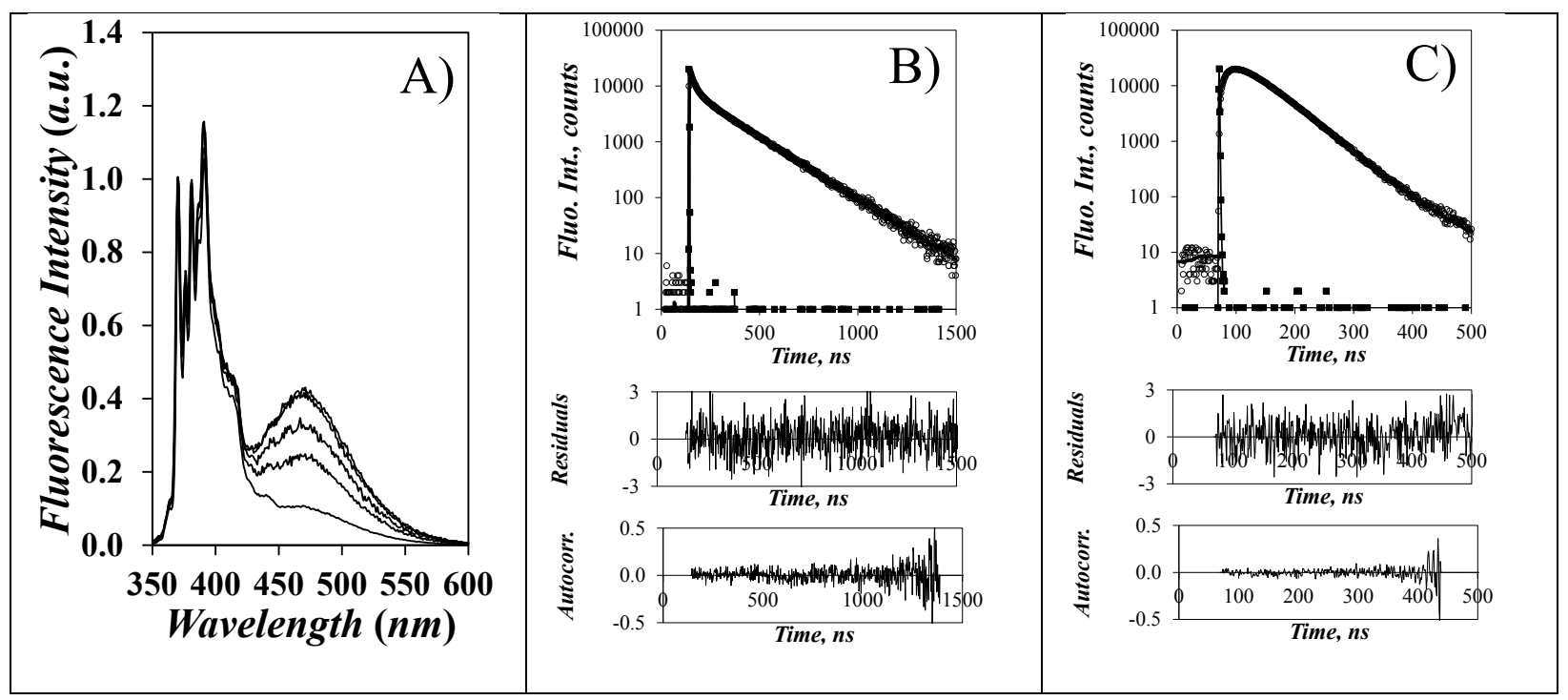

Figure 1. A) Fluorescence spectra of $50 \mathrm{mM}$ SDS without salt. From bottom to top: $[\mathrm{Py}]=0.14$, $0.31,0.41,0.51$, and $0.56 \mathrm{mM}$. Example of fit with globmis10ebg of the fluorescence decays of B) the monomer and C) the excimer in a micellar solution of $50 \mathrm{mM}$ SDS in water without salt containing $0.56 \mathrm{mM}$ pyrene, $\chi^{2}=1.05$.

The data obtained from fitting the monomer decays with miscatbbg and from fitting the monomer and excimer decays globally with globmis10ebg are first discussed. The average number of pyrenes per micelle $<n>$ obtained with both programs increased linearly with increasing pyrene 
concentration but yielded a slightly positive intercept. This non-zero intercept was due to residual impurities in the SDS sample, probably heavy metal counterions that quenched the pyrene monomer and artificially increased $\langle n>$ by a small constant value at all pyrene concentrations. For instance, the intercept equaled $0.11 \pm 0.03$ for the solutions prepared without salt $([\mathrm{NaCl}]=0 \mathrm{M})$, a small value that was nevertheless significantly higher than zero. After subtracting the intercept from the $<n>$ values, the straight lines obtained in Figure 2B passed though the intercept. $k_{\mathrm{q}}$ retrieved with the miscatbbg analysis program was also plotted as a function of pyrene concentration in Figure 2A. As the pyrene concentration increased, the SDS micelles uptook more pyrene molecules and $\langle n\rangle$ increased linearly with increasing pyrene concentration for all salt concentrations in Figure 2B. The increase in $<n>$ in Figure 2B did not affect the $k_{\mathrm{q}}$ value however since $k_{\mathrm{q}}$ represents the rate constant of excimer formation in a micelle containing one excited and one ground-state pyrene, and is thus independent of pyrene concentration. The linear relationship between $\langle n>$ and pyrene concentration was expected from Equation 4 that relates $<n>$ to the SDS and pyrene concentrations. Since the CMC of SDS at different salt concentrations had been determined earlier ${ }^{14}$ and since the pyrene and SDS concentrations were known, the slope of the straight lines obtained for each pyrene concentration yielded $N_{\text {agg }}$ which was plotted as a function of salt concentration in Figure 2D. The $N_{\mathrm{agg}}$ values increased with increasing salt concentration indicating micellar growth as is well-known in the literature. ${ }^{14,24}$ The $N_{\text {agg }}$ values shown in Figure 2D agreed really well with those obtained earlier by fitting the monomer decays alone. ${ }^{14}$ The global Micelle Model analysis of the monomer and excimer decays yielded the same $N_{\text {agg }}$ values in Figure 2D showing that including the excimer decay in the analysis did not affect the experimental results.

$$
<n>=\frac{[P y]}{[S D S]-C M C} \times N_{a g g}
$$




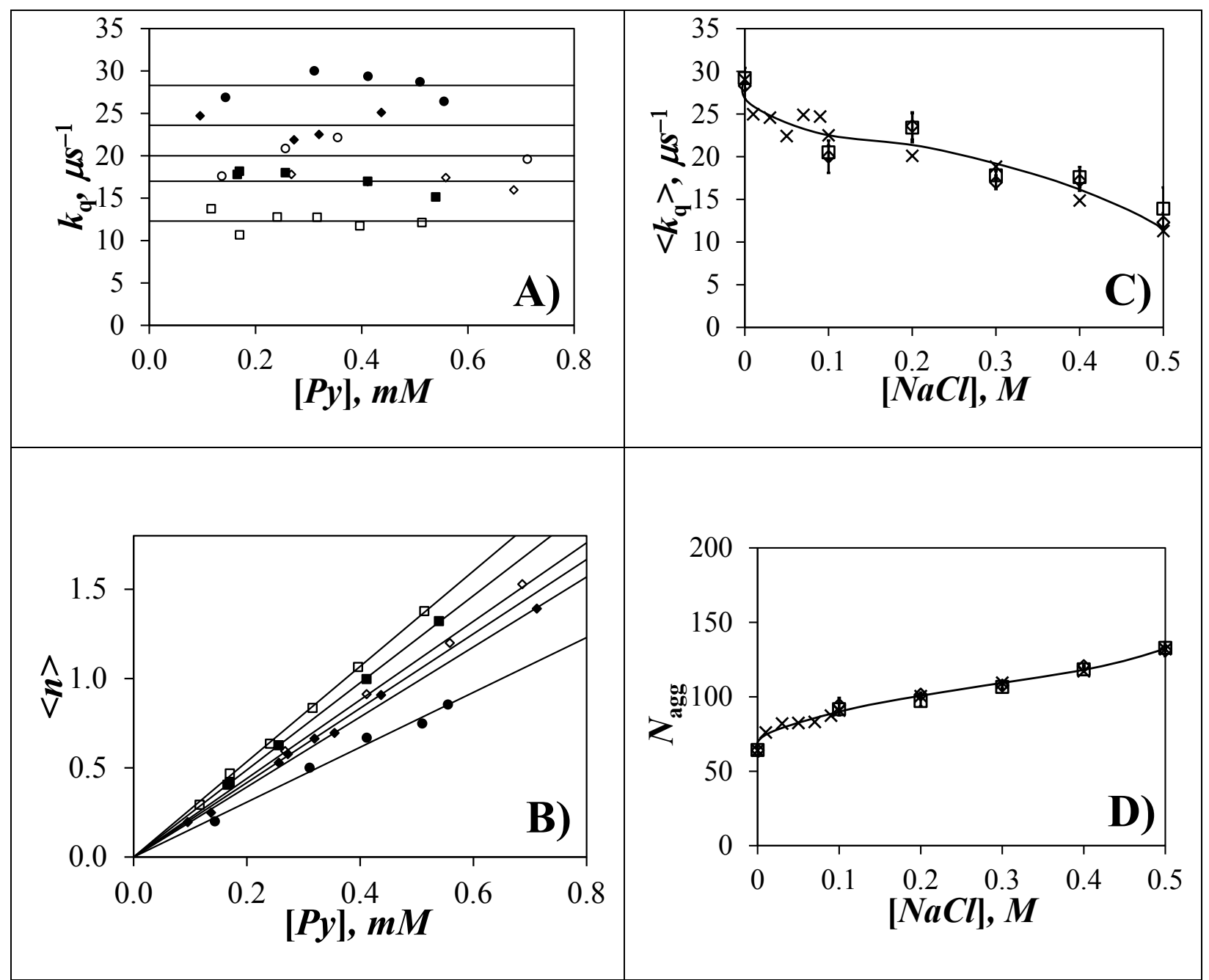

Figure 2. Plots of A) $k_{\mathrm{q}}$ and B) $<n>$ obtained from miscatbbg as a function of pyrene concentration.

$\mathrm{NaCl}$ concentrations equal $(\mathbf{O}) 0.0 \mathrm{M},(\mathbf{O}) 0.1 \mathrm{M},(>) 0.2 \mathrm{M}, \boldsymbol{\diamond}) 0.3 \mathrm{M}, \boldsymbol{\square}) 0.4 \mathrm{M}$, and $(\square)$

0.5 M. Plots of C) $<k_{\mathrm{q}}>$ and D) $N_{\text {agg }}$ as a function of $\mathrm{NaCl}$ concentration. $(\nabla)$ results from miscatbbg, $\square$ ) results from globmis 10ebg, $(\times)$ results from Ref. \#14. Lines in 2C and D have no physical meaning and are meant to guide the eye.

As mentioned earlier, the $k_{\mathrm{q}}$ values plotted in Figure 2A remained constant with pyrene concentration for a given salt concentration, within experimental error. The $k_{\mathrm{q}}$ values were averaged over all pyrene concentrations obtained at a same salt concentration to yield $<k_{\mathrm{q}}>$ which 
was plotted as a function of salt concentration in Figure $2 \mathrm{C} . \quad<k_{\mathrm{q}}>$ was found to decrease with increasing salt concentration, a consequence of the increased micellar size and viscosity of the micellar interior. ${ }^{14,24}$ Similar trends were obtained when the values of $k_{\mathrm{q}}$ and $<n>$ retrieved with globmis10ebg were plotted as a function of pyrene concentration (data not shown). Furthermore the same $<k_{\mathrm{q}}>$ and $N_{\text {agg }}$ were obtained in Figures $2 \mathrm{C}$ and D when the fluorescence decays were fitted with miscatbbg or globmis10ebg, respectively, thus demonstrating that both analyses were equivalent.

The lifetimes $\tau_{\mathrm{E} 0}$ and $\tau_{\mathrm{EL}}$ for the two excimers retrieved from the fits with globmis $10 \mathrm{ebg}$ equaled $44 \pm 5$ ns and $73 \pm 15$ ns, respectively. Two excimer species are often encountered when excimer formation occurs in restricted geometries as is often the case in PyLMs ${ }^{6,13}$ or, in the present study, SDS micelles. ${ }^{14}$ The lifetimes $\tau_{\mathrm{E} 0}$ and $\tau_{\mathrm{EL}}$ were typical of pyrene excimers generated in an organic medium ${ }^{25}$ such as the interior of the SDS micelles. Their combined contribution $f_{\text {agg }}$ $\left(=f_{\mathrm{E} 0}+f_{\mathrm{EL}}\right)$ equaled $0.025 \pm 0.010$ reflecting very little aggregation between the pyrene molecules located in the SDS micelles. The low level of aggregation between pyrenes is a consequence of the apolar environment generated by the micellar interior which is reflected by the $I_{1} / I_{3}$ ratio equal to $0.99 \pm 0.04$ averaged over 38 fluorescence spectra representing a medium that can properly solvate the pyrene molecules.

The monomer and excimer decays were then fitted globally with sumegs $34 \mathrm{bg}$ according to the MFA. The fits obtained with the MFA were equally good as with the global analysis of the monomer and excimer decays with globmis10ebg for the Micelle Model, as shown in Figure S1 in SI. The two lifetimes of the excimer obtained with sumegs $34 \mathrm{bg}$ equaled $44 \pm 4$ ns and $66 \pm 13$ ns for $\tau_{\mathrm{E} 0}$ and $\tau_{\mathrm{EL}}$, respectively. Within experimental error, these values are similar to those retrieved from the decay analysis with globmis10ebg. The level of pyrene aggregation was small 
with $f_{\text {agg }}$ equal to $0.026 \pm 0.010$, which, within experimental error, matched what was obtained with globmis10ebg.

Information about the type of probability describing the distribution of the pyrene molecules in the SDS micelles is stored in the monomer decays. In particular, MFA of the decays yields the molar fraction $f_{\text {Mfree }}\left(=\left[P y_{\text {free }}\right]_{\mathrm{o}} /\left(\left[P y_{\text {diff }}\right]_{\mathrm{o}}+\left[P y_{\text {free }}{ }^{*}\right]_{\mathrm{o}}\right)\right.$ of single pyrenes that are isolated in micelles (see Equation 1). In turn, $f_{\text {Mfree }}$ equals the probability of exciting a micelle containing a single pyrene. Taking into account the fact that the probability of exciting a micelle containing $i$ pyrenes is proportional to the probability of having $i$ pyrenes in a micelle multiplied by $i, f_{\text {Mfree }}$ would equal $\exp (-<n>)$ if the pyrene molecules were to distribute themselves randomly among the micelles according to a Poisson distribution. In turn, this hypothetical equality between $f_{\text {Mfree }}$ and $\exp (-<n>)$ would lead to Equation 5 which implies that if the pyrene molecules were to distribute themselves according to a Poisson distribution, a linear relationship would be expected between the pyrene concentration and $-\operatorname{Ln}\left(f_{\mathrm{Mfree}}\right)$.

$$
-\operatorname{Ln}\left(f_{\text {Mfree }}\right)=<n>=\frac{[P y]}{[S D S]-C M C} \times N_{\text {agg }}
$$

This linear relationship was indeed observed in Figure $3 \mathrm{~A}$ where $-\operatorname{Ln}\left(f_{\mathrm{Mfree}}\right)$ increased linearly with increasing pyrene concentration, thus demonstrating that pyrene molecules distribute themselves among the micelles according to a Poisson distribution. This demonstration is analogous to that used to establish that quenchers distribute themselves among micelles according to a Poisson distribution by showing a linear relationship between the logarithm of the fluorescence intensity of tris(bipyridine) ruthenium chloride dyes bound to SDS micelles as a function of 9methylanthracene quencher concentration. ${ }^{26}$ In fact, equating $-\operatorname{Ln}\left(f_{\text {Mfree }}\right)$ for $<n>$ yielded $N_{\text {agg }}$ by 
analyzing the slopes of the lines in Figure 3A with Equation 5. The $N_{\text {agg }}$ values obtained from the MFA matched very closely the $N_{\text {agg }}$ values obtained from the Micelle Model as shown in Figure $3 \mathrm{~B}$.

Further support of the excellent agreement between $-\operatorname{Ln}\left(f_{\text {Mfree }}\right)$ and $\langle n\rangle$ obtained, respectively, with the MFA and Micelle Model is shown in Figure 4 where $f_{\text {Mfree }}$ was plotted as a function of $\exp (-<n>)$ for the 28 monomer and 28 excimer fluorescence decays acquired in this study. A perfect linear relationship was obtained between the two quantities demonstrating their equivalence.
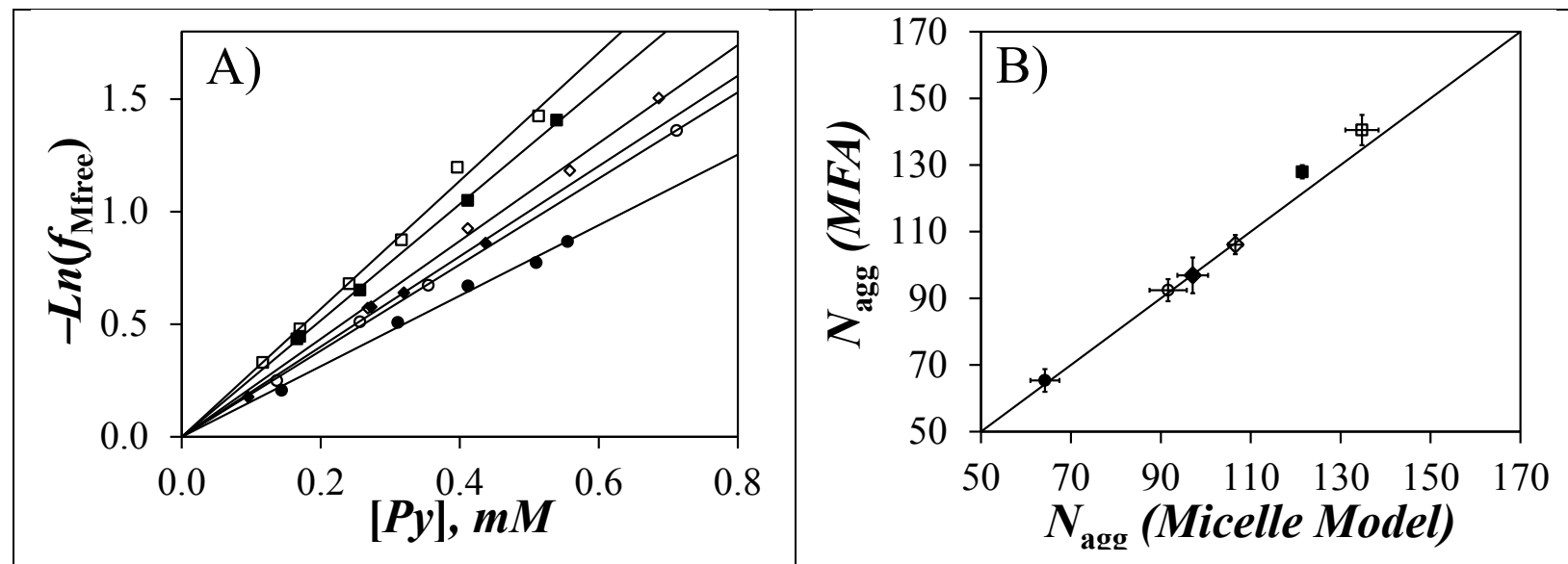

Figure 3. Plot of $\mathrm{A})-\operatorname{Ln}\left(f_{\text {Mfree }}\right)$ obtained from the MFA as a function of pyrene concentration and B) $N_{\text {agg }}$ obtained with the MFA as a function of $N_{\text {agg }}$ obtained with the Micelle Model. $\mathrm{NaCl}$ concentrations equal $(\bullet) 0.0 \mathrm{M},(\mathbf{O}) 0.1 \mathrm{M},(\boldsymbol{\nabla}) 0.2 \mathrm{M},(\boldsymbol{\diamond}) 0.3 \mathrm{M}, \boldsymbol{\square}) 0.4 \mathrm{M}$, and $(\boldsymbol{\square}) 0.5$ M.

Having established that the molar fraction $f_{\text {Mfree }}$ equaled the Poisson probability of having one pyrene per SDS micelle which corresponds to those pyrene molecules that are isolated and cannot form excimer, the rest of the decay described by the sum of exponentials corresponding to the front part of Equation 1 had to be related to the rate constant $k_{\mathrm{q}}$ that is being used in Equation 
3 for the Micelle Model. As it turns out, expanding Equation 3 into a sum of exponentials yielded Equation 6 where the first term represents the micelles that host a single pyrene while the other terms describe those micelles containing more than one pyrene that can form excimer.

$$
\begin{aligned}
{\left[P y^{*}\right](t)=\left[P y^{*}\right]_{o} \times\left[e^{-<n>} \frac{<n>^{0}}{0 !} \exp \left(-\left(0 \times k_{q}+\tau_{M}^{-1}\right) t\right)+\right.} \\
e^{-<n>} \frac{<n>^{1}}{1 !} \exp \left(-\left(1 \times k_{q}+\tau_{M}^{-1}\right) t\right)+ \\
e^{-<n>} \frac{<n>^{2}}{2 !} \exp \left(-\left(2 \times k_{q}+\tau_{M}^{-1}\right) t\right)+\ldots .
\end{aligned}
$$

Integration of the upper terms of Equation 6 yielded the average rate constant of pyrene excimer formation in SDS micelles given by Equation 7 .

$$
<k>=\frac{\sum_{i=1}^{\infty} \frac{<n>^{i}}{i !}}{\sum_{i=1}^{\infty} \frac{<n>^{i}}{i !} \frac{1}{i k_{q}+\tau_{M}^{-1}}}-\frac{1}{\tau_{M}}
$$




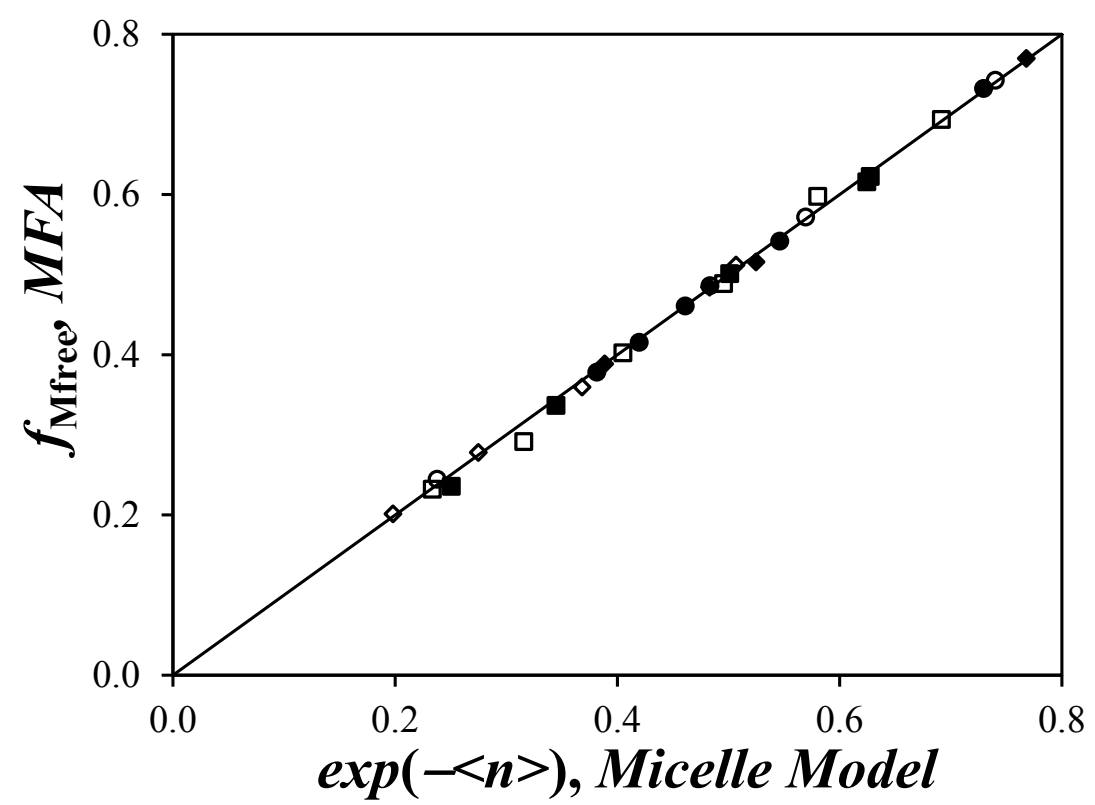

Figure 4. Plot of $f_{\text {Mfree }}$ obtained from the MFA and $\exp (-<n>)$ where $<n>$ was obtained from the Micelle Model. $\mathrm{NaCl}$ concentrations equal $0.0 \mathrm{M},(\mathbf{O}) 0.1 \mathrm{M},(>) 0.2 \mathrm{M},(\diamond) 0.3 \mathrm{M}$ $0.4 \mathrm{M}$, and ( $) 0.5 \mathrm{M}$.

In Equation $7,<n>$ equals $-\operatorname{Ln}\left(f_{\mathrm{Mfree}}\right)$ as demonstrated in Figure $4, \tau_{\mathrm{M}}$ is the lifetime of the pyrene monomer which is determined from the analysis of the fluorescence decays (see Table S4), and $<k>$ is obtained according to Equation 2 for the MFA. Consequently, Equation 7 contained a single unknown, $k_{\mathrm{q}}$. The rate constant $k_{\mathrm{q}}$ was determined by using a Golden Section Search ${ }^{20}$ to optimize the value of $k_{\mathrm{q}}$ that would best match $<k>$ determined form the MFA. Since the contributions to Equation 7 of the terms with $i>10$ were infinitely small, the summations for Equation 7 were conducted up to $i=20$. The resulting $k_{\mathrm{q}}$ value obtained from the MFA parameters was then plotted as a function of $k_{\mathrm{q}}$ obtained from the Micelle Model in Figure 5. The agreement between the $k_{\mathrm{q}}$ values obtained independently from both methodologies was rather good. 


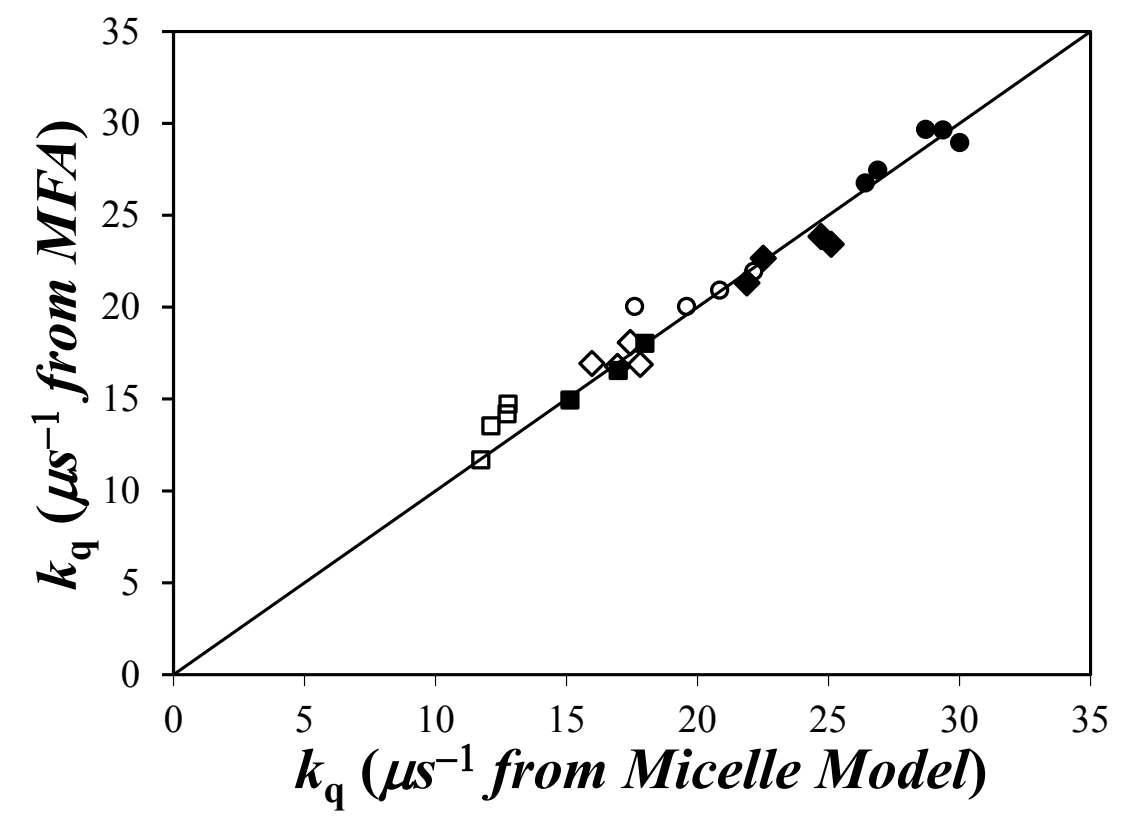

Figure 5. Plot of $k_{\mathrm{q}}$ obtained from the MFA and $k_{\mathrm{q}}$ obtained from the Micelle Model. $\mathrm{NaCl}$

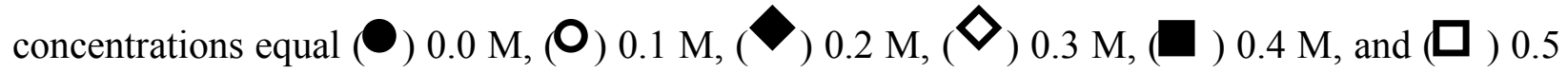
M.

Consequently, the trends shown in Figures 3-5 demonstrate that without making any assumption about the manner in which excimer formation proceeds between pyrenes randomly distributed among SDS micelles, the same dynamic (with $k_{\mathrm{q}}$ ) and structural (with $N_{\mathrm{agg}}$ ) information can be obtained with the MFA as with the Micelle Model. By adding the characterization of SDS micelles to an already extensive list of macromolecular systems to have been characterized by studying PEF with the MFA, ${ }^{6,13}$ the present study further expands the versatility and breadth of applicability of the MFA. 


\section{CONCLUSIONS}

Up to this day, the MFA has been solely applied to the characterization of PyLMs in solution. ${ }^{6,13}$ The present study departs from these earlier works by employing the MFA to characterize how PEF was generated in SDS micelles loaded with pyrene molecules. Compartmentalization of the pyrene molecules forming excimer in the confined geometry of SDS micelles was well described by the MFA. The parameters of interest retrieved from the MFA were $<k>$ and $f_{\text {Mfree }}$ which were found to be directly related to the parameters $k_{\mathrm{q}}$ and $<n>$ obtained from the MM. This meant that $f_{\text {Mfree }}$ and $<k>$ could be employed to gain, respectively, the same structural (in terms of $N_{\text {agg }}$ ) and dynamic information about SDS micelles as could be obtained from a MM analysis yielding $<n>$ and $k_{\mathrm{q}}$. However, while the Micelle Model makes several physical assumptions about the process of pyrene excimer formation inside the micelles (excimer formation in a micelle containing one excited pyrene and $i$ ground-state pyrenes takes place with a rate constant $i \times k_{\mathrm{q}}$ ) and the distribution of the pyrene molecules among the micelles which assumes Poisson statistics, the MFA made no such assumptions. The MFA reached the conclusion that the pyrene molecules distribute themselves among the SDS micelles according to Poisson statistics from the linear relationship that existed between $-\operatorname{Ln}\left(f_{\text {Mfree }}\right)$ and the pyrene concentration (Figure $\left.3 \mathrm{~A}\right)$. That the MFA was capable of reaching this conclusion came from its ability to retrieve $f_{\text {Mfree }}$ with very good accuracy

as has been shown in earlier publications. ${ }^{27}$ Furthermore the fact that despite its absence of assumptions, the MFA retrieved the same structural and dynamic information as the Micelle Model further strengthens the claim made earlier ${ }^{6,13}$ that the MFA can yield such information for any type of macromolecular system where PEF results from dynamic encounters between pyrene labels.

\section{ACKNOWLEDGMENTS}


The authors thank NSERC for generous funding.

\section{SUPPORTING INFORMATION}

Derivation of the Micelle Model equation for pyrene in SDS micelles, time-dependent expressions for the excimer concentration according to the Micelle model and MFA, examples of decay fit with the MFA, and parameters retrieved from the decay fits.

\section{REFERENCES}

1. Lakowicz, J. R. Principles of Fluorescence $3^{\text {rd }}$ Ed., Springer, New York, 2006.

2. Stryer, L.; Haugland, R. P. Energy Transfer: A Spectroscopic Ruler. Proc. Natl. Acad. Sci. 1967, 58, 719-726.

3. Bruscale, M.; Schuler, B.; Samori, B. Single-Molecule Studies of Intrinsically Disordered Proteins. Chem. Rev. 2014, 114, 3281-3317.

4. Doose, S.; Neuweiler, H.; Sauer, M. Fluorescence Quenching by Photoinduced Electron Transfer: A Reporter for Conformational Dynamics of Macromolecules. ChemPhysChem 2009, 10, 1389-1398.

5. Winnik, M. A. End-to-End Cyclization of Polymer Chains. Acc. Chem. Res. 1985, 18, 7379.

6. Duhamel, J. New Insights in the Study of Pyrene Excimer Fluorescence to Characterize Macromolecules and their Supramolecular Assemblies in Solution. Langmuir 2012, 28, 6527-6538.

7. Farhangi, S.; Duhamel, J. Long Range Polymer Chain Dynamics Studied by Fluorescence Quenching. 2016, 49, 6149-6162. 
8. Wilemski, G.; Fixman, M. Diffusion-controlled intrachain reactions of polymers. II results for a pair of terminal reactive groups. J. Chem. Phys. 1974, 60, 878-890.

9. Stals, P. J. M.; Li, Y.; Burdynska, J.; Nicolay, R.; Nese, A.; Palmans, A. R. A.; Meijer, E. W.; Matyjaszewski, K.; Sheiko, S. S. How Far Can We Push Polymer Architectures? J. Am. Chem. Soc. 2013, 135, 11421-11424.

10. Mathew, A.; Siu, H.; Duhamel, J. A Blob Model to Study Chain Folding by Fluorescence. Macromolecules 1999, 32, 7100-7108.

11. Duhamel, J. Polymer Chain Dynamics in Solution Probed with a Fluorescence Blob Model. Acc. Chem. Res. 2006, 39, 953-960.

12. Siu, H.; Duhamel, J. Comparison of the Association Level of a Hydrophobically Modified Associative Polymer Obtained from an Analysis Based on Two Different Models. J. Phys. Chem. B 2005, 109, 1770-1780.

13. Duhamel, J. Global Analysis of Fluorescence Decays to Probe the Internal Dynamics of Fluorescently Labeled Macromolecules. Langmuir 2014, 30, 2307-2324.

14. Fowler, M.; Hisko, V.; Henderson, J.; Casier, R.; Li, L.; Thoma, J.; Duhamel, J. DiPyMe in SDS Micelles - Artefacts and their Implications on Micellar Properties. Langmuir 2015, 31, 11971-11981.

15. Lopez, F.; Cuomo, F.; Ceglie, A. ; Ambrosone, L. ; Palazzo, G. Quenching and Dequenching of Pyrene Fluorescence by Nucleotide Monophosphates in Cationic Micelles. J. Chem. Phys. $B$ 2008, 112, 7338-7344.

16. Cuomo, F.; Palazzo, G.; Ceglie, A. ; Lopez, F. Quenching Efficiency of Pyrene Fluorescence by Nucleotide Monophosphate in Cationic Micelles. J. Phochem. Photobiol. A: Chem. 2009, 202, 21-27. 
17. Vethamuthu, M. S.; Almgren, M.; Mukhtar, E.; Bahadur, P. Fluorescence Quenching Studies of the Aggregation Behaviour of the Mixed Micelles of Bile Salts and Cetyltrimethylammonium Halides. Langmuir 1992, 8, 2396-2404.

18. Vasilescu, M.; Angelescu, D.; Almgren, M.; Valstar, A. Interactions of Globular Proteins with Surfactants Studied with Fluorescence Probe Methods. Langmuir 1999, 15, 2635-2643.

19. Alargova, R. G.; Kochijashky, I. I.; Sierra, M. L. ; Zana, R. Micelle Aggregation Numbers of Surfactants in Aqueous Solutions: A Comparison between the Results from Steady-state and Time-Resolved Fluorescence Quenching. Langmuir 1998, 14, 5412-5418.

20. Press, W. H.; Flannery, B. P.; Teukolsky, S. A.; Vetterling, W. T. Numerical Recipes. The Art of Scientific Computing (Fortran Version); Cambridge University Press: Cambridge, $\mathrm{p}$ 82, 1992.

21. Kalyanasundaram, K.; Thomas, J. K. Environmental Effects on Vibronic Band Intensities in Pyrene Monomer Fluorescence and their Application in Studies of Micellar Systems. J. Am. Chem. Soc. 1977, 99, 2039-2044.

22. Dong, D. C.; Winnik, M. A. The Py Scale of Solvent Polarities. Solvent Effects on the Vibronic Fine Structure of Pyrene Fluorescence and Empirical Correlations with ET and $Y$ Values. Photochem. Photobiol. 1982, 35, 17-21.

23. Dong, D. C.; Winnik, M. A. The Py Scale of Solvent Polarities. Can. J. Chem. 1984, 62, $2560-2565$.

24. Ranganathan, R.; Peric, M.; Medina, R.; Garcia, U.; Nales, B. L.; Almgren, M. Size, Hydration, and Shape of SDS/Heptane Micelles Investigated by Time-Resolved Fluorescence Quenching and Electron Spin Resonance. Langmuir 2001, 17, 6765-6770.

25. Birks, J. B. Photophysics of Aromatic Molecules; Wiley: New York, 1970; p 301. 
26. Turro, N. J.; Yekta, A. Luminescent Probe for Detergent Solutions. A Simple Procedure for Determination of the Mean Aggregation Number of Micelles. J. Am. Chem. Soc. 1978, 100, 5951-5952.

27. Chen, S.; Duhamel, J.; Bahun, G.; Adronov, A. Effect of Fluorescent Impurities in the Study of Pyrene-Labeled Macromolecules by Fluorescence. J. Phys. Chem. B 2011, 115, 99219929.

\section{Table of Content}

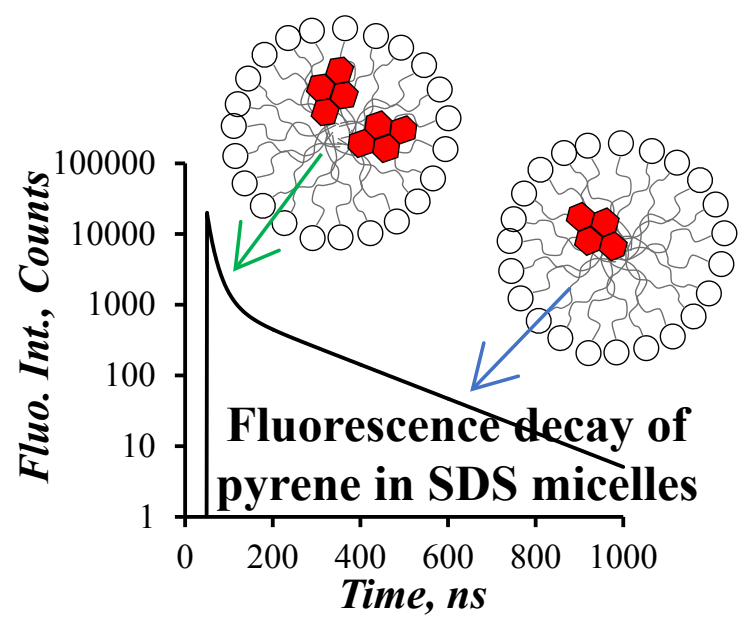

